upus nephritis is a common manifestation of systemic lupus erythematosus (SLE), and the current lack of reliable prognostic biomarkers for active renal disease can impede prompt management, causing some patients to progress to end-stage organ failure. The results of a study published in Rheumatology suggest that measurement of urinary neutrophil gelatinase-associated lipocalin (NGAL, also known as lipocalin 2) levels is a better predictor of lupus nephritis than other widely used laboratory measures of disease activity.

Renal involvement in patients with SLE is usually diagnosed on the basis of urinalysis, elevated serum levels of antidouble stranded (ds) DNA antibodies and erythrocyte sedimentation rate, and reduced $\mathrm{C} 3$ and $\mathrm{C} 4$ complement levels, as well as scores on indices such as the Systemic Lupus Erythematosus Disease Activity Index (SLEDAI). However, none of these tests is highly specific or sensitive for renal flare. Patients with evidence of active renal disease might undergo a biopsy, which can more accurately determine the presence of a renal flare, but the invasive nature of this technique and the tendency of lupus nephritis to recur bring the long-term safety of this approach to question.

It was against this background that Rubinstein et al. hoped to identify an improved prognostic biomarker for lupus nephritis in patients with SLE and a history of renal disease. "One of the major interests in our laboratories is investigating the renal pathogenicity of anti-DNA antibodies in patients with SLE; that is, understanding the mechanism by which anti-DNA antibodies are involved in lupus nephritis," explains Chaim Putterman, one of the study's researchers. "Several years ago, we found that pathogenic anti-DNA antibodies induce the expression of multiple genes in

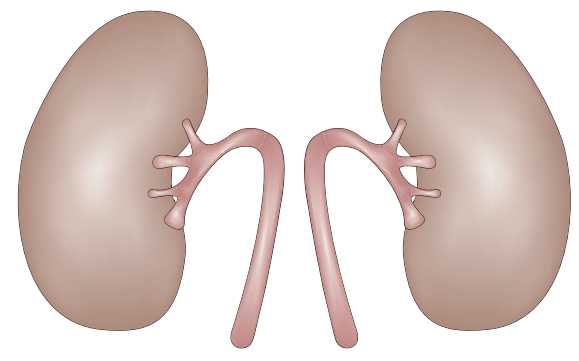

kidney cells in vitro and in vivo, including NGAL. Around the same time, other investigators found that kidney NGAL is markedly upregulated in response to a variety of insults to the kidney, and in fact is a sensitive and early urine marker in several types of human disease." In a study conducted in 2007, Putterman and colleagues showed that patients with lupus nephritis had higher urinary NGAL levels than SLE patients without lupus nephritis and controls. Based on these results, the investigators now sought to determine whether urinary NGAL could be used to identify the future risk of nephritis, as had been demonstrated in 2009 by Hinze et al. in their study of children with SLE.

Renal disease activity and flare status were determined by scores on the SLEDAI and in 107 patients with SLE, recruited from two centers in the USA. Patients were evaluated at two visits, and 25 patients had biopsy-proven nephritis. Using a random effects model, the researchers showed that high urinary NGAL levels could predict renal disease activity in all patients with SLE (odds ratio [OR] for each standard deviation increase 1.70), as well as in the subgroup with biopsy-proven nephritis (OR 4.63). By contrast, anti-dsDNA antibody titers and complement levels did not to seem to correlate with the onset of nephritis activity. Furthermore, high NGAL levels at the first evaluation visit successfully predicted increases in the renal, but not in the nonrenal, components of the SLEDAI index at the second visit, and biomarker levels were not associated with the level of proteinuria. These findings suggest that urinary NGAL levels are specific for renal disease rather than reflecting higher levels of general disease activity or protein excretion.

An analysis of the area under the receiver operating curve for the previous visit's NGAL level and renal flare identified a cut-off value for urinary NGAL of $13.6 \mathrm{ng} / \mathrm{ml}$, which provided a sensitivity and specificity of $89 \%$ and $72 \%$, respectively. Using this cut-off, the researchers were able to successfully validate their results against a longitudinal cohort of 35 patients from the UK, of whom 15 had biopsy-proven lupus nephritis, despite disparities in the demographics and criteria used to diagnose renal disease between the two cohorts.

The role of urinary NGAL as a biomarker for lupus nephritis in adult patients with SLE needs to be confirmed in large, prospective studies, such as the one that is currently being undertaken by Putterman and colleagues. "We plan to evaluate the performance of NGAL relative to other putative novel biomarkers in a head-to-head comparison," says Putterman. "In addition, we will determine whether a combination of several biomarkers would have improved clinical utility, and actually translate into improved care of patients with SLE."

\section{Negin Nassabeh}

